\title{
Aujuhu al-Tasyabuhi wa al-Tanahuri Baina al-Lughataini al-Arabiyah wa al-Tamiliyah Ala Mustawa al-Ashwat

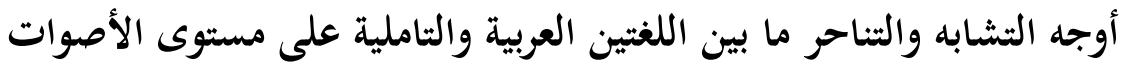

\section{MCS Shathifa}

shathifa@seu.ac.lk

Departement of Arabic Language

South Eastern University of Sri Lanka

\section{- Received: $24.07 .2020 \quad$ • Accepted: $20.11 .2020 \quad$ • Published online: 30.11 .2020}

Abstract: Language is every sound the people use to express their ideas and communicate with others. It is the basis of any language. Every language has its own unique systems in phonemic and orthographical symbols. According to this concept, Arabic is a Semitic language whereas Tamil is Indo -European language, there is no doubt that it is difficult to foreign language learners to identify the place of articulation of the unfamiliar sounds, its pronunciations and writing format. Among them are the Tamil - speaking Muslims who use and learn Arabic as a foreign language. They face difficulties in pronouncing and writing Arabic letters, proper nouns and term words due to the lack of clarity in the sounds between these two languages. This study try to identify similarities and differences between the sound of Arabic and Tamil languages. This research focus on descriptive and contrastive methods to analyze the data gathered from secondary data such as books and previous studies related to the topic. The result indicates that the Arabic letters are differ from Tamil letters in terms of their number, writing method and their shapes. And also, it has six vowels similar to the six vowels of Tamil language in pronunciation. But they differ in places of articulation and its aspects. Thus, Consonants of Arabic differ from Tamil consonants in pronunciation, places of articulation and IPA for them. Consequently, this study will support to determine the pronunciation of Arabic letters and read Arabic language in the best way.

Keywords: Language, Sounds, Arabic Language, Tamil Language 
الملخص: إن اللغة هي أصوات يعبر بها كل قوم عن آرائهم وأفكارهم، وهي أداة تساعد على الاتصال بالآخرين. وكل لغة تتركب من جوانب أربعة : الأصوات، الصرف، النحو ، المعنى. وأما الأصوات فهي أساس اللغة، ولكل لغة نظام صوتي خاص ورموز اصطلاحية. من خلال هذا

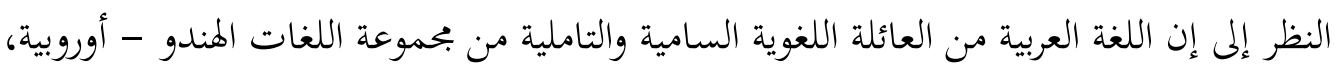
ومما لاشك فيه يصعب على متعلمي اللغة الأجنبية تحديد مخارج الأصوات الجحديدة ونطقها وكتابتها. ومنهم المسلمون الناطقون باللغة التاملية الذين يستخدمون ويتعلمون اللغة العربية كلغة أجنبية، فهم يواجهون المشقة في نطق وكتابة الحروف والأعلام والمصطلحات العربية لعدم الوضوح في أصوات اللغتين العربية والتاملية. فتركزت هذه الدراسة تحديد أصوات اللغتين العربية والتاملية، والكشف عن مواقع الشبه والخلاف الصوتي بين اللغتين. استخدمت هذه الدراسة المنهج الوصفي والتقابلي لتحليل المعلومات الثانوية التي حصلت عليها من الدراسات السابقة والكتب والمحلات والمقالات وماتتعلق بهذا الموضوع من الشبكات الإلكترونية. وفي ختام هذه الدراسة قد حددت الباحثة نتائج شتى، ومنها: إن الحروف العربية تتناحر عن الحروف التاملية من حيث عددها وطريقة كتابتها واختلاف أشكالها حسب المواقع في الكلمات. كما أن في اللغة العربية ستة أصوات صائتة وهي تتشابه ستة أصوات صائتة في اللغة التاملية حسب النطق ولكن هناك تناحرا بين صوائتهما من حيث المخرج والصفة، كما أن الصوامت العربية تتناحر كثيرا عن الصوامت التاملية من حيث النطق والمخارج والرموز الصوتية الدولية لها. وإن هذه الدراسة تساعد على المسلمين وغير المسلمين الناطقين باللغة التاملية في تحديد نطق الأصوات العربية وقراءة اللغة العربية بأحسن وجه في المستقبل بواسطة الوضوح بين أصوات هتين اللغتين. كلمات دلالية: اللغة ، الأصوات، اللغة العربية، اللغة التاملية 
Mohammed Cassim Sithy Shathifa

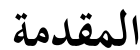

اللغة هي وسيلة التفاهم والتبادل، والهدف الرئيس لتعلم وتعليم اللغة هو التواصل والتبادل مع الآخرين. وبالنظر إلى هذا يتعلم الناس لغات متعددة لغة أولوية، أو ثانية، أو أجنبية. ومنهم المسلمون السريلانكيون الذين يتعلمون اللغة العربية لغة أجنبية من حيث كوها لغة القرآن الكريم ومحمد صلى الله عليه وسلم.

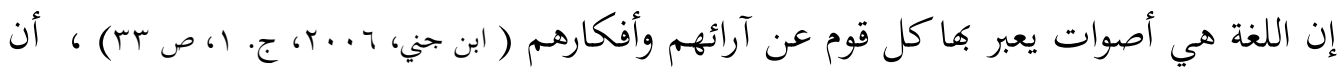
أصوات الكلام هي اللبنات التي تشيد منها الكلمة وأن الكلمات هي التي تشيد منها الجملة وأن الجمل هي اللبنات لتشيد الكلام، فإن الأصوات هي أساس البناء التركيبي ومن تم،

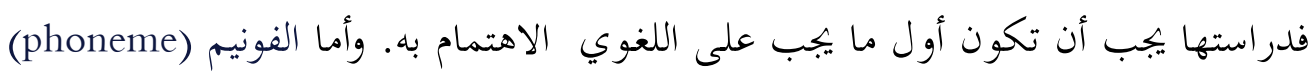
هو "أصغر وحدة لغوية صوتية بحردة تفرق بين كلمة وأخرى". وهو يعني صوتا لغويا، مثل:

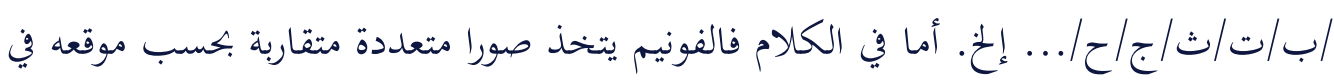
الكلمة وما يسبقه وما يلحقه من أصوات أخرى. فمثلا فونيم /ر/ في (رجع) يختلف نطقه قليلا

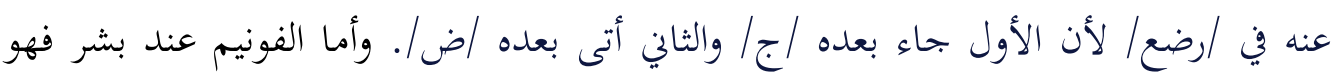

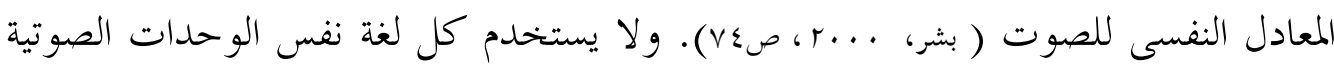
التي تستعملها لغة أخرى لكي تركب منها الكلمات، وإنما يستعمل كل لغة وحدات صوتية مختلفة، وهذه الوحدات الصوتية تسمى الفونيمات Phonemes (حلمي، 1990، ص rr). وقد أشار تروبتسكوى "Trubetzkoy" إلى هذا المفهوم للفونيم على أنه النماذج الصوتية التي لها قدرة على تمييز الكلمات وأشكاها أو الأنماط الصوتية المستقلة التي تميز الحلدث الكلامى المعين

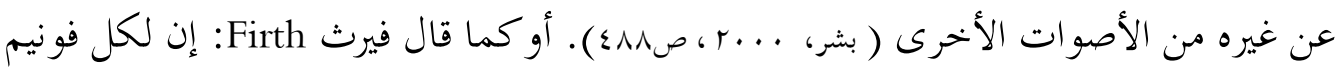

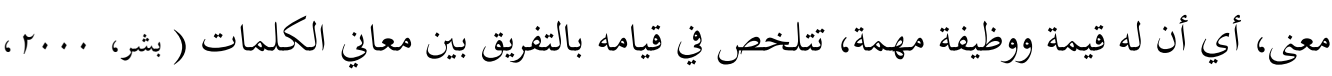
ص99 ). وهذا هو الرأي المقبول عند بشر.

وعلى هذا، فالنون في " نام" هي فونيم يشترك مع الفونيمات الأخرى في الكلمة لتحديد

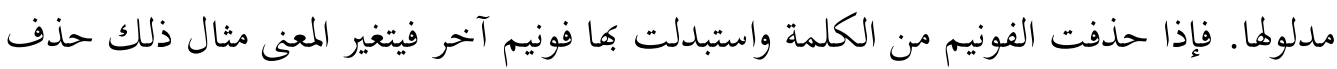
فونيم الصاد من صام واستبداله بفونيم القاف فتصبح الكلمة قام. ففي اللغة التاملية يوجد 
Mohammed Cassim Sithy Shathifa

تغاير في المعنى بتغير الفونيم مثلا: بين الكلمتين Бாடு(Kadu), நாடு(Nadu) وبين

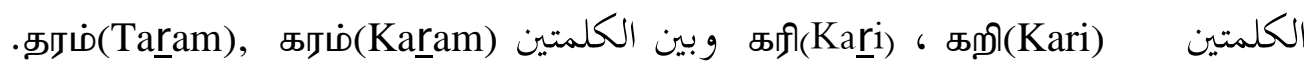
الفونيمات إذن كأصوات لما سماتحا الخاصة، قادرة على التمييز بين الكلمات في معظم اللغات. بل هى قادرة على التمييز من ناحية ترتيبها أيضا في صلب الكلمة مثلا بين الكلمتين بلغ، وغلب لهب وبين الكلمتين رجع، وجعر. ويتضح ذلك في التقابل بين الكلمات التاملية - Karam)கரம் (tasam)தசம்-(satham)சதம்,(taram)தரம்-( ㅁatham)ரதம்،(raham)ரகம் المثال، حيث تتكون هنا ثلاث كلمات مختلفة من نفس الفونيمات ولكن بترتيب مختلف. وهو يؤدي إلى حدوث كلمات جديدة. وحين الحدديث عن الفونيم في اللغة العربية فإننا نشير إلى أن الأصوات المفردة عبارة عن بحموعة من الفونيمات، فحرف ( تاء) يمكن أن تحل محلها الدال فالتاء فونيم، والدال فونيم، و يتضح ذلك في التقابل بين الفونيم التاملية فحرف (k) ه يمكن أن تحل محل (h) ஹஹ. ولكنهما فونيمان مختلفان. وكذلك التقابل في العربية بين الفتحة والضمة، فكلمة (كَرَم) اسم في العربية، ولكن (كرَّر ،

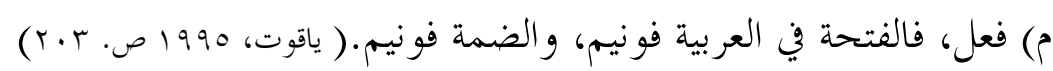

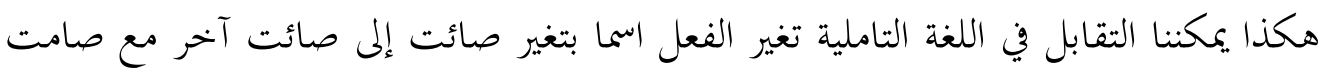

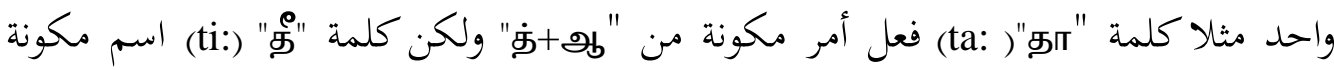

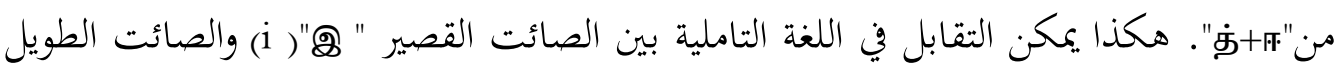

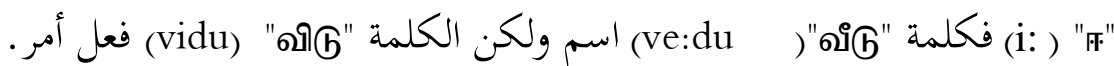
إلى جانب أن للفونيم الخاصيتين التين اتفق عليهما العلماء، فلا بد أن يشار إليهما بالبنان

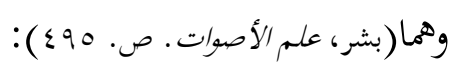

1- - أن الفونيمات جزء من نظام اللغة المعينة وأها تختلف في عددها وخواصها من لغة

$$
\text { إلى أخرى. }
$$


Mohammed Cassim Sithy Shathifa

r- أن البحث في الفونيمات ينصرف بتمامه إلى اللغة المنطوقة، إذ هي وحداتها الصوتية. أما اللغة المكتوبة فوحداتا هي الرموز الموضوعة لترجمة المنطوق، وتسمى

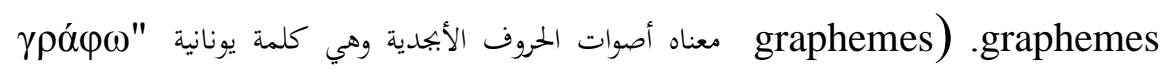
"write"-gráphō

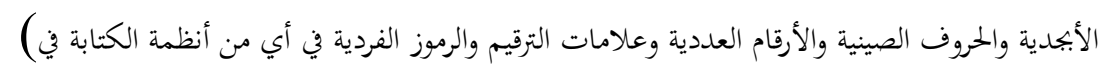

كما أن للفونيم قسمين : أحدهما فونيم قطعي (Segmental) والآخر فونيم فوقطعي

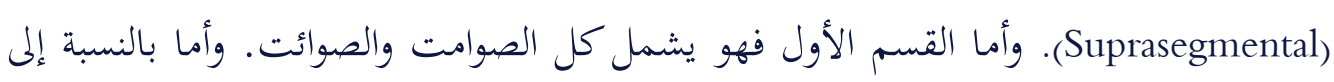
القسم الثاني فهو يتمثل في كل من الفاصل (Juncture) والنغم (Pitch) والنبرة (Stress) وطول

الصوت Word length)

أما الفونيمات اللغوية محدودة في كل اللغات، وعلى هذا هناك تشابه بين غالبية الأصوات في نفس الوقت يوجد اختلاف في بعضها بين اللغات. ومما لاشك فيه أنه يصعب على متعلمي اللغة

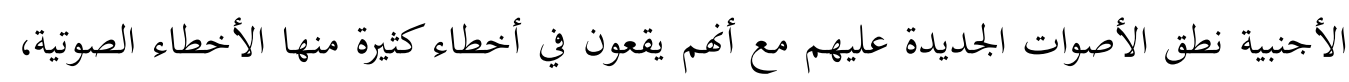
ويلعب نقل الأنظمة الصوتية من اللغة الأصلية أثناء تعلم لغة أجنبية دورا سلبياً في الوقوع في أخطاء صوتية.

إن اللغة التاملية هي اللغة المحلية لأغلبية المسلمين بسريلانكا. واللغة العربية لغة أجنبية لمسلمي سريلانكا. وبالإضافة فالعربية من العائلة اللغوية السامية والتاملية من مجموعة اللغات الهندوأوروبية، وأن أوجه التناحر بينهما أكثر من وجوه التشابه، ولعدم الوضوح في تمييز التشابه والتناحر بين أصوات هتين اللغتين يواجه المسلم الناطق في سريلانكا الصعوبات في الختيار الأحرف المناسبة عند نطق وكتابة النصوص والمصطلحات والأعلام العربية بالحروف التاملية، وربما أفم لا يحسنون

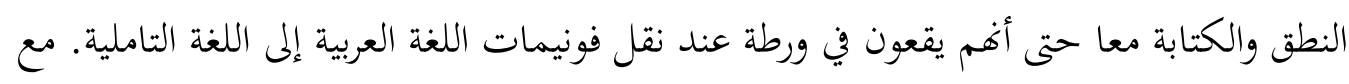

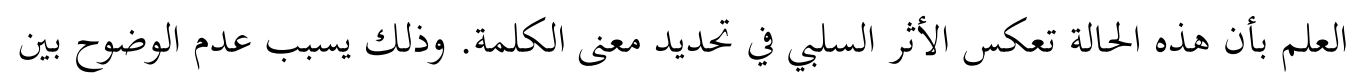
أصوات اللغتين العربية والتاملية.

وعلى هذا تريد هذه الدراسة تسليط الضوء على تحديد أوجه التشابه والاختلاف بين النظامين

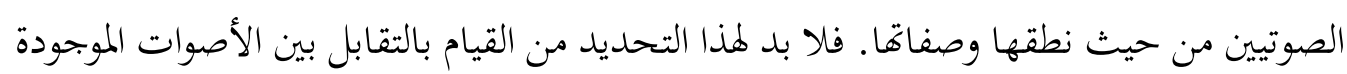


في اللغتين العربية كلغة منشودة والتاملية كلغة أم لأن المنهج التقابلي يساعد على التنبؤ بالمشكلات النطقية ووصف ما يتعلق منها بأداء المتكلمين بلغة معينة عندما يدرسون لغة أخرى. كما إن هذه الدراسة تساعد على ترقية المعرفة بعلم الأصوات عامة وعلم أصوات اللغة العربية والتاملية خاصة مع أها تساعد أيضا على تطوير نطق وكتابة السليمين عند من لا يجيدون العربية من الناطقين بالتاملية.

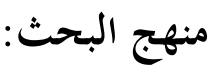

قد نشأ حديثًا المنهج التقابلي في إطار المدرسة الوصفية لدراسة اللغة وخدمة أهداف تربوية في جانب علم اللغة التطبيقي في بحالات متعددة: أهمها بحال تعليم اللغات والترجمة، فثمة صعوبات تصادف من يتعلمون لغة أخرى (كلغة ثانية). بالإضافة إلى لغتهم الأم، وهذه الصعوبات نابتحة عن الاختلاف الموجود بين نظام اللغة الأم ونظام اللغة الثانية. بناء على هذا، يستفيد هذا البحث لتحقيق أهدافه المذكورة من المنهج الوصفي والتقابلي لتقابل الأصوات الموجودة في اللغتين العربية والتاملية.

إن اللغتين العربية والتاملية مختلفتان من حيث عائلتهما اللغوية. ومن هنا، يتقابل هذا البحث أوجه الشبه والخلاف بين أصوات اللغتين عموما. وثم يناقش عن الأصوات الصائتة والأصوات الصامتة فيهما مع ذكر التشابهات والاختلافات بينهما خلال المنهج التقابلي، كما أن اللغتين تتضمن الأصوات الصائتة والأصوات الصامتة فهي تتقابل نطقا وكتابة لتحديد الفروق الدقيقة بين الأصوات.

تستخدم المصادر الثانوية لمعرفة النظريات عن اللغتين العربية والتاملية، وتاريخ نشأتما، ومضموناها الصوتية من الأصوات الصائتة والأصوات الصامتة ومخارجها وصفاتما. وهذه النظريات تسهل الدراسة التقابلية بينهما. بناء على هذا، تحصل المعلومات الثانوية من الكتب والبحوث السابقة والمقالات الإلكترونية وغيرها من الوثائق. 
Mohammed Cassim Sithy Shathifa

المناقشة والنتائج

كل لغة تتميز عن غيرها من اللغات بمجموعة من الفروقات من أبرزها عدد الحروف وحركاتا

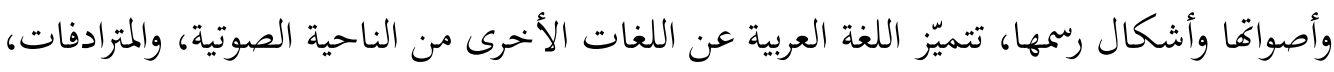
والوضوح، وشدة الارتباط بين الصوت والمعنى في كلماتما، والاشتقاق، والإعراب، والتغير في الدلالات بتغيير بنية الكلمات. إن البناء الصوتي في اللغة العربية يختلف كثيراً عما يقابله في اللغة ولئ

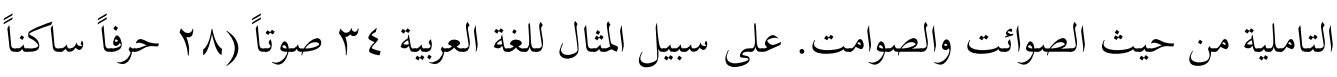
و T حروف علة: ثلاثة قصيرة وهي الفتحة والكسرة والضمة وثلاثة ممدودة). وحروف العلة القصيرة تكتب حركات باستخدام التشكيل المناسب إما فوق أو تحت الحرف الساكن. وهذه الحركات اختيارية. وأيضا تتميز اللغة العربية عن غيرها من اللغات بمجموعة من الحروف الحلقية التي تميزها مثل: العين، والحاء، والغين، والخناء، والهاء، والهمزة، والحروف المطبقة مثل الصاد، والضاد، والظاء، وحرف القاف. ومن لا يعرف مخارج النطق الصحيحة للحروف العبية ومواقع الشبه والخلاف بين

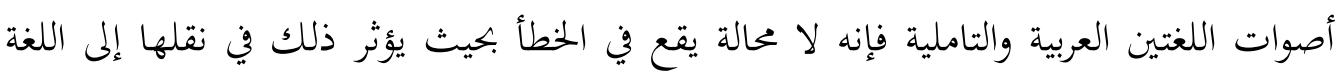
الهدف ويؤدي إلى تعدد طرق وأساليب الكتابة. وينتج عن ذلك عدة استخدامات للكلمة

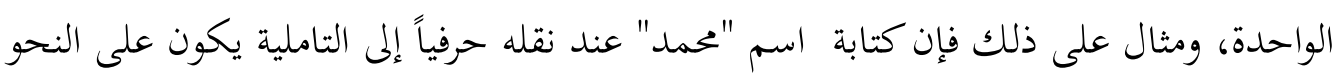
التالي ”ؤفض் أحرف العلة عليه، كما نلاحظه كالتالي (முஹம்மத், முகம்மத்,மொஹம்மத்) ومثال آخر ف فإن كتابة كلمة "زكاة" عند نقله حرفيا إلى التاملية يكون على النحو التالي " العلة عليه، كما في المثال التالي (சகாத்,ஸகாத்) . وهنا نلاحظ من المثالين اختيار الحرفين المختلفين من اللغة التاملية لصوت واحد في اللغة العربية واختيار الحرف الواحد من اللغة التاملية للصوتين المختلفين في اللغة العربية. فإن هذه الدراسة تعالج قضية التشابة والتناحر بين أصوات

$$
\begin{aligned}
& \text { اللغتين تحت العناوين الثلاثة : } \\
& \text { 1- أوجه الشبه والخلاف بين أصوات اللغتين عموما } \\
& \text { r- أوجه الشبه والخلاف بين الصوائت العربية والتاملية }
\end{aligned}
$$


Mohammed Cassim Sithy Shathifa

$$
\begin{aligned}
& \text { r- أوجه الشبه والخلاف بين الصوامت العربية والتاملية } \\
& \text { 1- أوجه الثبه والخلاف بين أصوات اللغتين عموما }
\end{aligned}
$$

> إن الحروف العربية تكتب من اليمين إلى اليسار في حين أن الحروف التاملية تكتب من

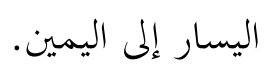

إن الحروف العربية تختلف أشكالها حسب اختلاف مواقعها في الكلمات كما تختلف

أشكال الصوائت التاملية حسب اختلاف مواقعها في الكلمات ولكن الصوامت التاملية

$$
\text { لا تختلف أشكالها حسب اختلاف مواقعها في الكلمات. }
$$

إن الحروف العربية كلها يقع في كل من المواقع في الكلمات من حيث الأول والأوسط والآخر في حين أن الحروف التاملية كلها لا يقع في كل من المواقع في الكلمات.

r - أوجه التشابه والتناحر بين صوائت اللغتين نطقا وكتابة

وتتفق اللغة العربية مع اللغة التاملية في الصوائت قصيرة وطويلة. فالصوائت العربية القصيرة الثلاث تقابل بالصوائت التاملية القصيزة الثلاث كما يلي:

$$
\begin{aligned}
& \text {-الفتحة القصيرة تقابل (1) /a/) } \\
& \text { /lu/ الضمة القصيرة تقابل ( } \\
& \text { |i/ الكسرة القصيرة تقابل ( - }
\end{aligned}
$$

وأما الصوائت العربية الطويلة الثلاث فهي تقابل بالصوائت التاملية الطويلة الثلاث حسبما يأي: /a:/ الفتحة الطويلة تقابل ( ) /u:/ الضمة الطويلة تقابل ( )

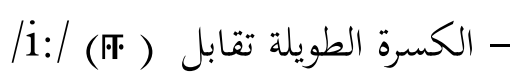


Mohammed Cassim Sithy Shathifa

وجه الخلاف بينهما:

ومع الإتفاق في صوائت كلتا اللغتين يوجد اختلاف في بعض الأمور أيضا :

أ - إن عدد الصوائت في اللغة التاملية اثنا عشر في حين أن عددها في العربية ستة فقط، لذلك

يصعب الحصول على الرموز البديلة في اللغة العربية لبعض من الصوائت التاملية

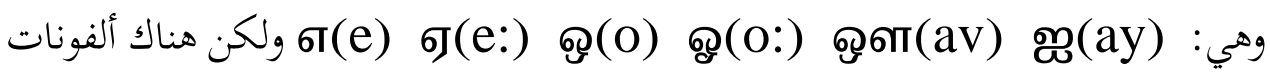

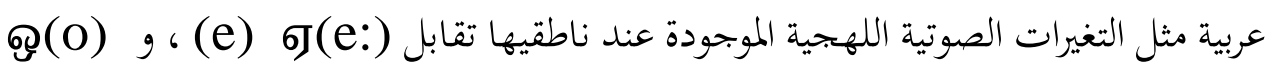

(Shu'ayb,1993, P. 782) •ஓ(O:)

ب- تختلف صوائت اللغة العربية عن التاملية من حيث مخارج الأصوات والصفات كالآتي في

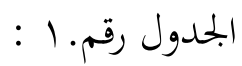

- الصوائت الأمامية في التاملية أربعة في حين أها في العربية ثلاثة فقط.

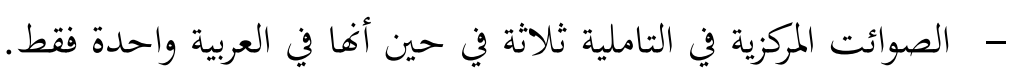
- الصوائت الخلفية في التاملية خمسة في حين أنها في العربية اثنتان فقط.

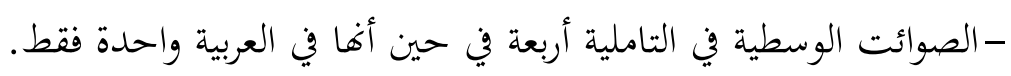
- الصوائت المنخفضة في التاملية ثلاثة في حين أغا في العربية واحدة فقط. ج- أما الفتحة القصيرة فهي تختلف عن الصائت التاملي (1) ( ن نطقا حسب المخرج

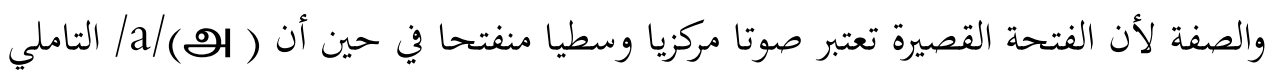
يعتبر صوتا مركزيا منخفضا منفتحا.

وكذلك الفتحة الطويلة تختلف عن الصائت التاملي ( م /a: / نطقا حسب المخرج والصفة

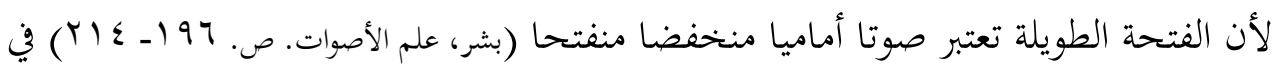

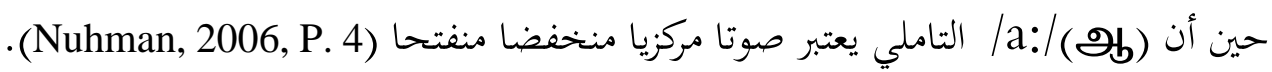
والجدول التالي يعطي الصورة الحقيقية عن أوجه التشابه والتناحر بين صوائت اللغتين. 


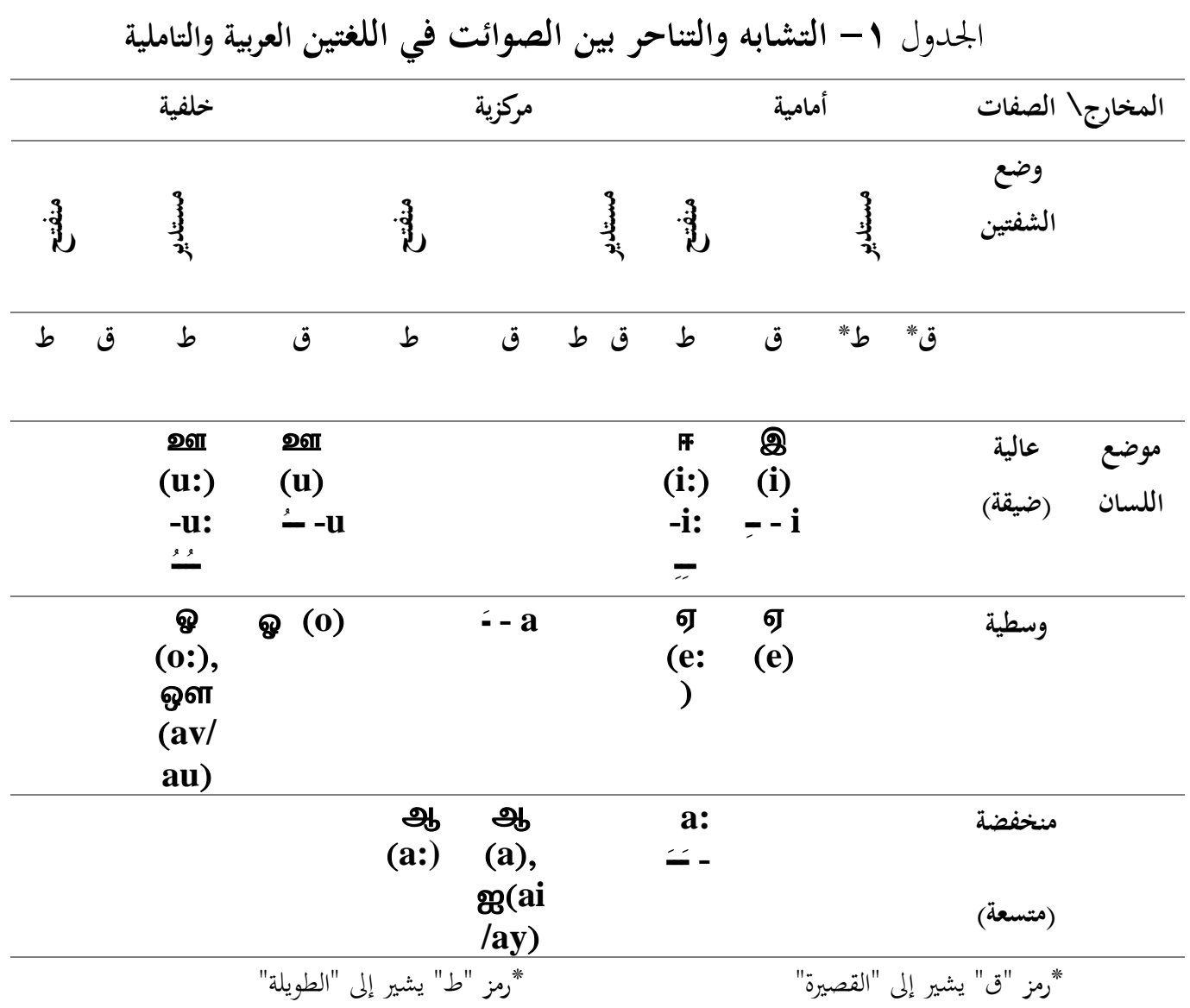

ب- أوجه التشابه والتناحر بين صوامت اللغتين نطقا وكتابة

كما أن هناك قضايا تتفق وتختلف كلتا اللغتين في الصوائت وكذلك أن هناك قواسم مشتركة ومفترقة في مسئلة الصوامت وهي كالتالي :

وجه الشبه : إن وجه الشبه في بعض من صوامت اللغتين العربية والتاملية يظهر في الشكلين : الشكل الأول يكون حسب النطق مع اتفاق المخارج والرموز الموضوعة لها ـ وأما الشكل الثاني فهو حسب النطق فتط مع اختلاف المخارج والرموز الموضوعة لها. 
فالصوامت المشتركة وفق النطق مع اتفاق المخارج والرموز الموضوعة لها في اللغتين كما يلي:

$\begin{array}{cccc}\mathrm{m} & \text { ம } & \mathrm{m} & - \\ \mathrm{s} & \text { ச } & \mathrm{s} & - \\ \mathrm{k} & \text { க } & \mathrm{k} & -5 \\ \mathrm{y} & \text { ய } & \mathrm{y} & -\mathrm{s}\end{array}$

أما الصوامت المشتركة وفق النطق فتط مع اختلاف المخارج والرموز الموضوعة لها في اللغتين فهي كما يأني:

\begin{tabular}{|c|c|c|}
\hline$\underline{t}$ & த & $\mathrm{t}$ \\
\hline$n$ & ன & $\mathrm{n}$ \\
\hline 1 & ல & 1 \\
\hline V & வ & W \\
\hline$\underline{r}$ & $\mathfrak{M}$ & $\mathrm{r}$ \\
\hline$n_{n}^{n}$ & ந & $\mathrm{n}$ \\
\hline
\end{tabular}

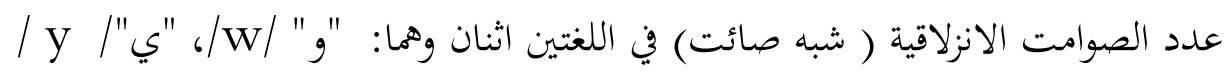
وجه التناحر:

تختلف الصوامت التاملية من الصوامت العربية في سبع مسائل وهي: أ- - عدد الصوامت في التاملية ثمانية عشر في حين أن عددها في العربية ثمانية وعشرون. ب- عدد مخارج الصوامت في التاملية ثمانية (Nuhman, 2006, P. 8) أما عددها في العربية أحد عشر مخرجا (عبد الله، مقدمة في علم الأصوات. ص. هـ). ومن الملاحظ أن لا توجد المخارج اللهوية والحلقية والحنجرية في النظام الصوتي للغة التاملية. 
Mohammed Cassim Sithy Shathifa

ج- عدم وجود البدائل العربية لثمان من الصوامت التاملية وهي (Shu’ayb,1993, P. 782) :

$\begin{array}{lrrr}\mathfrak{d} & \text { ட } & \mathrm{p} & \text { ப } \\ \mathfrak{n} & \text { ண } & \underline{r} & \text { ர } \\ \mathfrak{\eta} & \text { Ђ } & \tilde{\mathrm{n}} & \text { ஞ } \\ ! & \text { ள } & \underline{1} & \text { ழ }\end{array}$

د- عدم وجود البدائل التاملية لتسعة عشر صامتا من الصوامت العربية وهي:

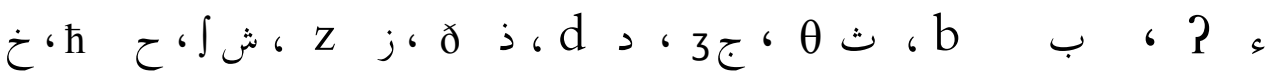

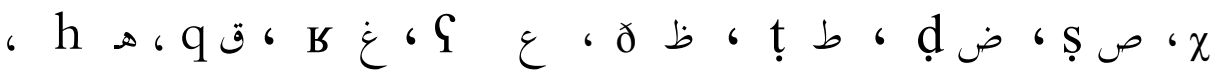

ف

هـ - إن من الصوامت العربية صوامت مزجية وهي "ج"/ $3 /$ مع العلم بأن هذا القسم لايوجد في

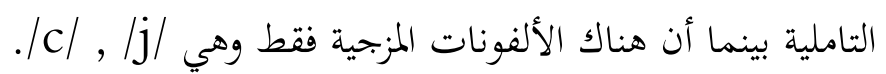

و - توجد في اللغة العربية صوامت بحهورة ومهموسة باعتبار صفاتما بينما هي في اللغة التاملية صوامت مهموسة فقط إضافة إلى الألفونات البمهورة.

ز- تتميز اللغة التاملية بكثرة وجود الأصوات الأنفية وهي في التاملية ستة في حين أنها في العربية

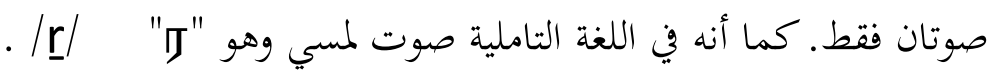

مع العلم بأن هناك في اللغة العربية ثمانية أصوات احتكاكية أما في اللغة التاملية فصوت واحد لهد

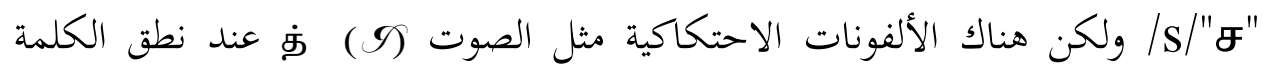

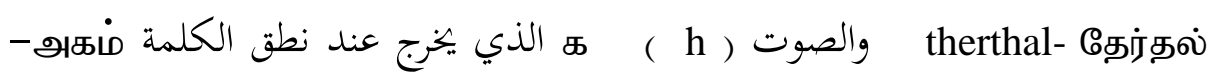
safam -சாபம் والصوت (م) ب الذي يظهر عند نطق الكلمة aham ح- ومن الصوامت التاملية الألفونات التي تقابل الأصوات العربية ومنها ما لا تقابل لها. $\begin{array}{rr}\text { الألفونات التي تقابل الأصوات العربية: } & \text { b }\end{array}$

ه h $\quad$ C J J 
Mohammed Cassim Sithy Shathifa

$$
\text { الألفونات التي لا تقابل الأصوات العربية: }
$$

$\begin{array}{rrrrrr}\text { pattam -பட்டம } & t & \text { L } & \text { patrai -பற்றை } & \mathrm{t} & \mathrm{m} \\ \text { paccai -பச்சை } & \mathrm{c} & \text { ச } & \text { param - படம் } & \mathrm{r} & \text { ட } \\ & & & \text { சங்கம் } & \mathrm{g} & \text { க }\end{array}$

إن في العالم لغات شتى، ولكن هناك تشاهما وتناحرا من حيث الأصوات والبنية والتراكيب والدلالة حسب الأسر اللغوية. من وجهة هذا النظر إن بين اللغتين العربية والتاملية تشاها وتناحرا من حيث الأصوات لأكما لغتين من أسرتين مختلفتين. فلابد لدارسي اللغة العربية لغة أجنبية أن يتضح بين أصوات هتين اللغتين. إن هذه الدراسة تساعد على المسلمين وغير المسلمين الناطقين باللغة التاملية في تحديد نطق الأصوات العربية وقراءة اللغة العربية بأحسن وجه في المستقبل بواسطة الوضوح بين أصوات هنين اللغتين.

إن الحروف العربية تتناحر عن الحروف التاملية من حيث عددها وطريقة كتابتها واختلاف أشكالها حسب المواقع في الكلمات. كما أن في اللغة العربية ستة أصوات صائتة وهي تتشابه ستة أصوات صائتة في اللغة التاملية حسب النطق ولكن هناك تناحرا بين صوائتهما من حيث المخرج والصفة. كما أن الصوامت العربية تتناحر كثيرا عن الصوامت التاملية من حيث النطق والمخارج والرموز الصوتية الدولية لها. من هذه الناحية، يقدم هذا البحث بعض التوصيات ومنها: لابد لدارسي اللغة أن يتضح في علم الأصوات، ويجب على المسلمين الناطقين باللغة التاملية معرفة التشابه والتناحر بين أصوات اللغتين العبية والتاملية، وتقديم التوعية عن التشابه والتناحر بين أصوات اللغتين العربية والتاملية للمعلمين والمتعلمين اللغة العربية في سريلانكا. 
Mohammed Cassim Sithy Shathifa

\section{المصادر والمراجع}

\section{المراجع العربية:}

ابن جني، أبو الفتح عثمان. (r 19 1 ) الخحصائص. القاهرة: المكتبة العلمية.

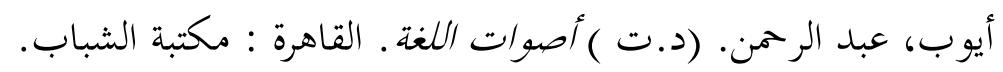

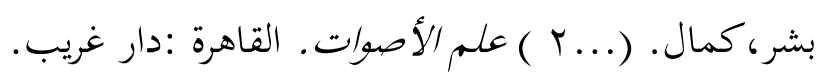

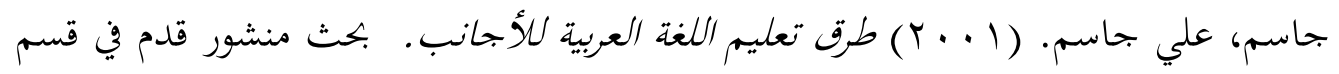

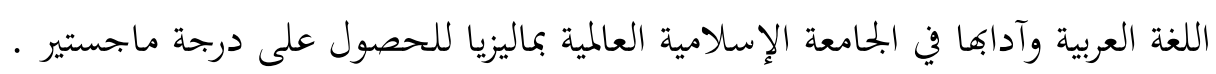
جلال،فؤاد علي و محمود، شوكت طه. (Y V V أثثر الاضطرابات الصوتية والنطقية في تعلم

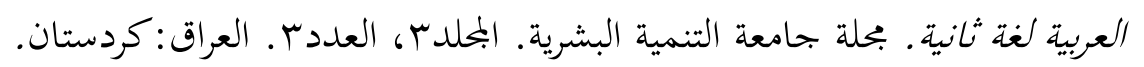

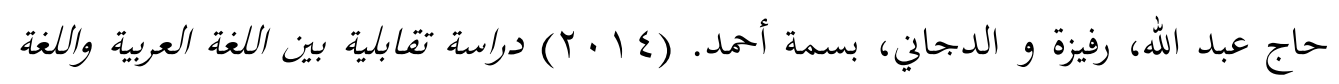
الملايوية : التعريف والتنكير نموذجا ـ ورقة بحثية منشورة في بحلة "دراسات" للعلوم الإنسانية والاجتماعية، المجلد إع، ملحق ا ـ عمان : الأردن - جامعة الأردن.

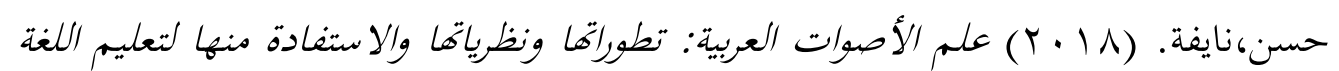

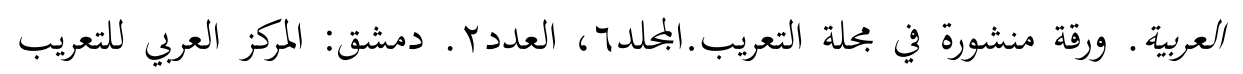

$$
\text { والترجمة والتأليف والنشر. }
$$

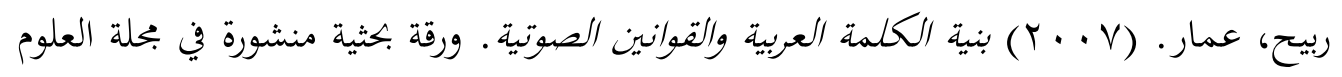

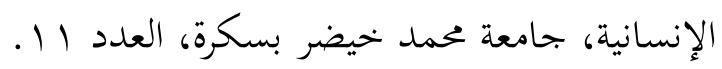

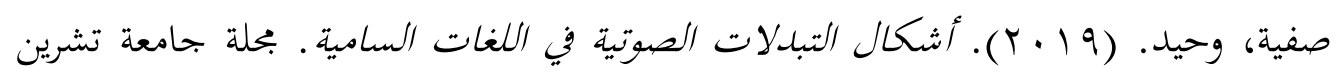

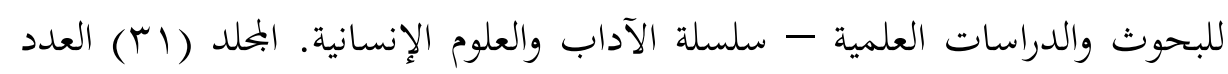

عبد الله، عادل. ( ع . . †) مقدمة في علم الأصوات. ط. (، الماليزيا : الجامعة الإسلامية العالمية. عودة، أبو الفتوح، (1910 ) )، قواعد نقل حروف الكلمات العربية إلى الحروف اللاتينية، بحلة المكتبات والمعلومات، العدد ب، السنة السادسة، المملكة العربية السعودية: دار المريخ. 


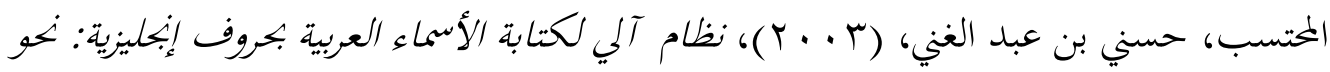

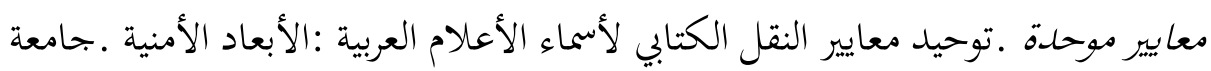

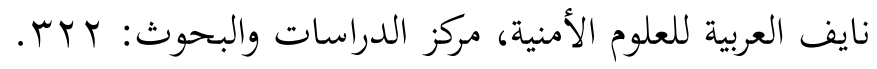

مسعود، عبد الرحيم. (1/ • †) دراسة تقابلية لبعض المظاهر الصوتية في اللغة العربية ولغة يوريا. بحلة الدراسات اللغوية والأدبية. العدد 9.

المسند، حمزة كريم و الدجاني، بسمة أحمد صدقي. (7 ( • ب) منهاج تعليم العربية للناطقين بغيرها

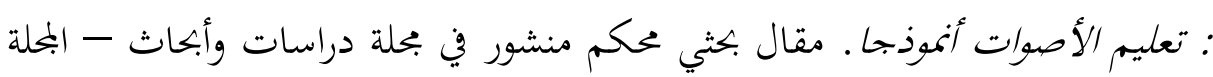

$$
\text { العربية في العلوم الإنسانية والاجتماعية. }
$$

مشتاق عباس معن وخالد جواد جاسم. (د.ت) ظاهرة تعلدد الأوجه النطقية في ضوء التغيرات فوق التركيبية : النبر والتنغيم والوقف. ورقة بحثية منشورة في دواة - بحلة فصلية محكمة

$$
\text { تعني بالبحوث والدراسات اللغوية. }
$$

المهنا، فيصل محمد، (T · . ب)، الأسما العربية بين الكتابة الصوتية والنتل الكتابي. الندوة العلمية للنقل الكتابي للأسماء بين اللغات "رومنة الأسماء العربية"، الرياض: مركز الدراسات

$$
\text { والبحوث - قسم الندوات والإلقاء العلميات. }
$$

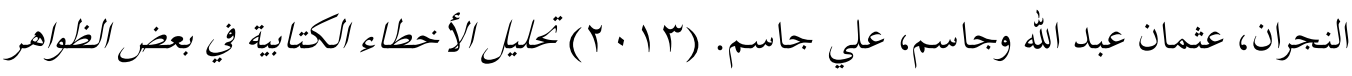
النحوية في كتابات الطلاب غير الناطقين بالعربية.بحث محكم منشور في المؤتم الدولي

الثامن للغة العربية، جامعة إمام بونجول الإسلامية الحكومية بادانج، إندونيسيا. نيدياساري، هنينديو فوتري. (9 ( ب ) تحليل الأخطاء الصوتية والاستغادة منها في تصميم الوسيلة التعليمية لمادة علم الأصوات في قسم تعليم اللغة العربية بجامعة جاكرتا الحكومية. رسالة ماجستير قدمت في قسم تعليم اللغة العربية. مالانج : جامعة مولانك مالك إبراهيم الإسلامية الحكومية. 
Mohammed Cassim Sithy Shathifa

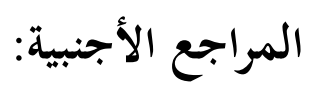

Karunakaran, K. (1997) Moliiyal. Chennai: Kumaran Pathippaham.

Karunakaran, K. (2000) Simplified grammar of Tamil. Chennai: Suvita publishers.

Nuhman, M.A. (2006) Adippatai Tamil Ilakkanam. Kalmunai: Readers Society.

Sanmukathas, A. (1982) Tamil Moli Ilakkana Iyalfukal. Jafna: Muthtamil veliyeettuk Kalakam.

Sanmuham Sivesekaran, C. (1993) Tamilum Ayalum. Chennai: South Asian Books.

Shu'ayb, Taika. (1993) Arabic, Arwi and Persian in Sarandib and Tamil Nadu. Madras: Imamul Arus Trust.

Seenivasan, S. (N.D) Ariviyal payanpattukkena tamil eluthurukkanathai $\begin{array}{llll}\text { mempaduthal; } & \text { Retrieved } & 09- & \text { 10- }\end{array}$ www.infitt.org/ti2000/papers/papersB.pdf . 